\title{
POSSIBILITIES AND LIMITATIONS OF USING SCHEDULING THEORY IN THE PROCESS INDUSTRY
}

J.Peter van Dam, University of Groningen, NL

Gerard J.C. Gaalman, University of Groningen, NL

Gerard Sierksma, University of Groningen, NL

\begin{abstract}
Scheduling in process industries is very complex, due to for example (partly) identical machines, shared resources, sequencedependent set-up times, flow shops with limited intermediate storage, and manual operations carried out off-line. Moreover, it is not possible to define one objective function. objectives of the scheduling are to obtain a feasible schedule, to meet due dates, to minimize the inventory, and to minimize additional costs such as costs of overtime and extra personnel.

In our view these scheduling problems contain elements of various lependent problems, as the assignment problem, the travelling salesman problem, and the flowshop scheduling problem. Although much literature can be found about these separate scheduling problems, there is not much attention for the integral problem.

In practice we observed a scheduling hierarchy of two levels. The type of decisions taken at each level, varies among the different scheduling situations. In making a schedule, the schedulers use rules of thumb. There are many other ways to make a schedule too. We think that before developing algorithms, the complex scheduling situation must be analyzed and ordered. Therefore, we define scheduling characteristics which describe the situations found in practice. The scheduling algorithms we develop, are based on these characteristics. This will by demonstrated by means of an application in a practical case.
\end{abstract}

